\title{
Lattice Points in the Newton Polytopes of Key Polynomials
}

\author{
NEIL J.Y. FAN, PETER L. GUO, SIMON C.Y. PENG, SOPHIE C.C. SUN
}

Abstract. We confirm a conjecture of Monical, Tokcan and Yong on a characterization of the lattice points in the Newton polytopes of key polynomials.

\section{Introduction}

Key polynomials $\kappa_{\alpha}(x)$ associated to compositions $\alpha \in \mathbb{Z}_{\geq 0}^{n}$, also called Demazure characters, are characters of the Demazure modules for the general linear groups [1,2]. They are nonsymmetric polynomial generalizations of Schur polynomials. Key polynomials are intimately connected with other important polynomials in algebraic combinatorics. For example, every Schubert polynomial is a positive sum of key polynomials (see for example Lascoux and Schützenberger [11, Reiner and Shimozono [16]), every key polynomial is a positive sum of Demazure atoms (see for example Haglund, Luoto, Mason and van Willigenburg [5], Lascoux and Schützenberger [10], Mason [13]). Moreover, $\kappa_{\alpha}(x)$ can be realized as a specialization of the nonsymmetric Macdonald polynomial $E_{\alpha}(x ; q, t)$, that is, $\kappa_{\alpha}(x)=E_{\alpha}(x ; q=\infty, t=\infty)$, see Ion [6].

This paper is concerned with the Newton polytope of $\kappa_{\alpha}(x)$. Given a polynomial

$$
f=\sum_{\alpha \in \mathbb{Z}_{\geq 0}^{n}} c_{\alpha} x^{\alpha} \in \mathbb{R}\left[x_{1}, \ldots, x_{n}\right],
$$

the Newton polytope of $f$ is the convex hull of the exponent vectors of $f$ :

$$
\operatorname{Newton}(f)=\operatorname{conv}\left(\left\{\alpha: c_{\alpha} \neq 0\right\}\right) \text {. }
$$

By definition, each exponent vector of $f$ is a lattice point in Newton $(f)$. If every lattice point in Newton $(f)$ is also an exponent vector of $f$, then we say that $f$ has saturated Newton polytope (SNP). The SNPness of polynomials has been investigated by Monical, Tokcan and Yong [14]. They [14, Conjecture 3.10] conjectured that key polynomials have the SNPness property. This conjecture was confirmed by Fink, Mészáros and St. Dizier [4]. It was also conjectured by Monical, Tokcan and Yong [14, Conjecture 3.13] and proven by Fan and Guo [3] that the vertices of $\operatorname{Newton}\left(\kappa_{\alpha}\right)$ can be generated by permutations in a lower interval in the Bruhat order.

Monical, Tokcan and Yong [14, Conjecture 3.11] further conjectured a characterization of the lattice points in $\operatorname{Newton}\left(\kappa_{\alpha}\right)$, or equivalently, of the exponent vectors of $\kappa_{\alpha}(x)$. The task of this paper is to prove this conjecture. Let $\alpha=\left(\alpha_{1}, \ldots, \alpha_{n}\right) \in \mathbb{Z}_{\geq 0}^{n}$. For $1 \leq i<j \leq n$, let $t_{i, j}(\alpha)$ be the composition obtained from $\alpha$ by interchanging $\alpha_{i}$ and $\alpha_{j}$, and let

$$
m_{i, j}(\alpha)=\alpha+e_{i}-e_{j}
$$

where $e_{k}(1 \leq k \leq n)$ is the standard coordinate vector. For a vector $\beta \in \mathbb{Z}_{>0}^{n}$, define $\beta \leq_{\kappa} \alpha$ if $\beta$ can be generated from $\alpha$ by applying a sequence of moves $t_{i, j}$ for $\alpha_{i}<\alpha_{j}$, and $m_{i, j}$ for $\alpha_{i}<\alpha_{j}-1$. 
Theorem 1.1 (Monical-Tokcan-Yong [14, Conjecture 3.11]). A vector $\beta$ is a lattice point in $\operatorname{Newton}\left(\kappa_{\alpha}\right)$ if and only if $\beta \leq_{\kappa} \alpha$. Equivalently, $\beta$ is an exponent vector of $\kappa_{\alpha}(x)$ if and only if $\beta \leq_{\kappa} \alpha$.

When the parts of $\alpha$ are weakly increasing, $\kappa_{\alpha}(x)$ equals the Schur polynomial $s_{\lambda}(x)$, where $\lambda$ is the partition obtained by rearranging the parts of $\alpha$ decreasingly, see for example Reiner and Shimozono [16]. In this case, the Newton polytope of $s_{\lambda}(x)$ is $\mathcal{P}_{\lambda}$, the permutohedron whose vertices are rearrangements of $\lambda$. A classical theorem due to Rado [15] states that for a partition $\mu, \mathcal{P}_{\mu} \subseteq \mathcal{P}_{\lambda}$ if and only if $\mu \unlhd \lambda$ in the dominance order. By Rado's theorem, it is easy to check that the lattice points of $\mathcal{P}_{\lambda}$ are the rearrangements of partitions $\mu$ with $\mu \unlhd \lambda$. On the other hand, it can be shown that when $\alpha$ is weakly increasing, $\beta \leq_{\kappa} \alpha$ if and only if $\beta$ is a rearrangement of some partition $\mu \unlhd \lambda$. This yields a proof of Theorem 1.1 for the case when $\alpha$ is weakly increasing.

The following corollary is a direct consequence of Theorem 1.1, which generalizes Rado's theorem from partitions to compositions.

Corollary 1.2. The Newton polytope Newton $\left(\kappa_{\beta}\right)$ of $\kappa_{\beta}(x)$ is contained in the Newton polytope $\operatorname{Newton}\left(\kappa_{\alpha}\right)$ of $\kappa_{\alpha}(x)$ if and only if $\beta \leq_{\kappa} \alpha$.

We also remark that Theorem 1.1 leads to a description of the lattice points in certain Bruhat interval polytopes. For two permutations $u \leq v$ in the Bruhat order, the Bruhat interval polytope $\mathrm{Q}_{u, v}$ is the convex hull of the permutations in the Bruhat interval $[u, v]$. Bruhat interval polytopes were introduced by Kodama and Williams [7], and their combinatorial properties were studied by Tsukerman and Williams [17]. When $\alpha=w$ is a permutation of $[n]=\{1,2, \ldots, n\}$, that is, the parts of $\alpha$ are rearrangements of $1,2, \ldots, n$, the Newton polytope Newton $\left(\kappa_{w}\right)$ coincides with $\mathbf{Q}_{w, w_{0}}$ [3, Corollary 1.3], where $w_{0}=$ $n \cdots 21$. Hence, when $\alpha=w$ is a permutation of $[n]$, Theorem 1.1 characterizes the lattice points in the Bruhat interval polytope $\mathbf{Q}_{w, w_{0}}$.

We prove Theorem 1.1 for any compositions by employing the realization of key polynomials as the dual characters of flagged Weyl modules associated to skyline diagrams, see Section 2. The structure of flagged Weyl modules has been used by Fink, Mészáros and St. Dizier [4] to prove the SNPness of Schubert polynomials and key polynomials. In Section 3, using the flagged Weyl module associated to the skyline diagram $D(\alpha)$ of $\alpha$, we encode the monomials appearing in $\kappa_{\alpha}(x)$ in terms of column-strict flagged fillings of $D(\alpha)$. We introduce operations on column-strict flagged fillings of $D(\alpha)$, which enable us to reflect the moves $t_{i, j}$ and $m_{i, j}$ from compositions to column-strict flagged fillings of $D(\alpha)$. This allows us to obtain a proof of Theorem 1.1 .

\section{Key polynomials and flagged Weyl modules}

Key polynomials can be defined using the Demazure operators $\pi_{i}=\partial_{i} x_{i}$. Here, $\partial_{i}$ is the divided difference operator, that is, given a polynomial $f(x) \in \mathbb{Z}\left[x_{1}, \ldots, x_{n}\right], \partial_{i}$ sends $f(x)$ to

$$
\partial_{i}(f(x))=\frac{f(x)-s_{i} f(x)}{x_{i}-x_{i+1}}
$$


where $s_{i} f(x)$ is obtained from $f(x)$ by exchanging $x_{i}$ and $x_{i+1}$.

If $\alpha$ is a partition (that is, the parts of $\alpha$ are weakly decreasing), then set $\kappa_{\alpha}(x)=x^{\alpha}$. Otherwise, choose $i$ such that $\alpha_{i}<\alpha_{i+1}$. Let $\alpha^{\prime}$ be the composition obtained from $\alpha$ by interchanging $\alpha_{i}$ and $\alpha_{i+1}$, namely, $\alpha^{\prime}=t_{i, i+1}(\alpha)$. Set

$$
\kappa_{\alpha}(x)=\pi_{i}\left(\kappa_{\alpha^{\prime}}(x)\right)=\partial_{i}\left(x_{i} \kappa_{\alpha^{\prime}}(x)\right) .
$$

The above definition is independent of the choice of the position $i$, since the Demazure operators satisfy the braid relations: $\pi_{i} \pi_{j}=\pi_{j} \pi_{i}$ for $|i-j|>1$, and $\pi_{i} \pi_{i+1} \pi_{i}=\pi_{i+1} \pi_{i} \pi_{i+1}$. For example, for $\alpha=(1,3,2)$, we have

$$
\begin{aligned}
\kappa_{(1,3,2)}(x) & =\pi_{1} \kappa_{(3,1,2)}(x)=\pi_{1} \pi_{2} \kappa_{(3,2,1)}(x)=\pi_{1} \pi_{2}\left(x_{1}^{3} x_{2}^{2} x_{3}\right) \\
& =x_{1}^{3} x_{2}^{2} x_{3}+x_{1}^{3} x_{2} x_{3}^{2}+x_{1}^{2} x_{2}^{3} x_{3}+x_{1}^{2} x_{2}^{2} x_{3}^{2}+x_{1} x_{2}^{3} x_{3}^{2} .
\end{aligned}
$$

In the remaining of this section, we briefly review the structure of flagged Weyl modules associated to diagrams of an $n \times n$ grid. In particular, the key polynomial $\kappa_{\alpha}(x)$ is equal to the dual character of the flagged Weyl module associated to the skyline diagram of $\alpha$. The flagged Weyl modules can be constructed by means of determinants [12. Here we follow the notation in [4].

A diagram $D$ is a collection of boxes of an $n \times n$ grid. We use $(i, j)$ to denote the box of the $n \times n$ grid in row $i$ and column $j$, where the row indices increase from top to bottom and the column indices increase from left to right. With this notation, a diagram $D$ can be written as an ordered list $D=\left(D_{1}, D_{2}, \ldots, D_{n}\right)$ of $n$ subsets of $[n]$, this is, $i \in D_{j}$ if and only of $(i, j)$ is a box of $D$. For example, the diagram in Figure 2.1 can be represented as $(\{1\}, \emptyset,\{1,2,3\},\{2,3\})$.

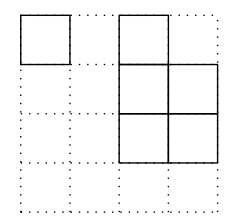

Figure 2.1: A diagram of a $4 \times 4$ grid.

Let $\operatorname{GL}(n, \mathbb{C})$ be the group of $n \times n$ invertible matrices over $\mathbb{C}$, and let $B$ be the subgroup consisting of the $n \times n$ upper-triangular matrices. Let $Y$ be the $n \times n$ uppertriangular matrix whose entries are indeterminates $y_{i j}$ where $i \leq j$. Denote by $\mathbb{C}[Y]$ the ring of polynomials in the variables $\left\{y_{i j}\right\}_{i \leq j}$. The group $\operatorname{GL}(n, \mathbb{C})$ acts on $\mathbb{C}[Y]$ (on the right) as follows. Given a matrix $g \in \mathrm{GL}(n, \mathbb{C})$ and a polynomial $f(Y) \in \mathbb{C}[Y]$, define

$$
f(Y) \cdot g=f\left(g^{-1} Y\right) .
$$

For two diagrams $C=\left(C_{1}, \ldots, C_{n}\right)$ and $D=\left(D_{1}, \ldots, D_{n}\right)$, write $C \leq D$ if $C_{j} \leq D_{j}$ for every $1 \leq j \leq n$, where $C_{j} \leq D_{j}$ means that $\left|C_{j}\right|=\left|D_{j}\right|$ and for $1 \leq k \leq\left|C_{j}\right|$, the $k$-th least element of $C_{j}$ is less than or equal to the $k$-th least element of $D_{j}$. The flagged Weyl module $\mathcal{M}_{D}$ associated to a diagram $D$ is a $B$-module defined by

$$
\mathcal{M}_{D}=\operatorname{Span}_{\mathbb{C}}\left\{\prod_{j=1}^{n} \operatorname{det}\left(Y_{D_{j}}^{C_{j}}\right): C \leq D\right\},
$$


where, for two subsets $R$ and $S$ of $[n], Y_{S}^{R}$ denotes the submatrix of $Y$ with row indices in $R$ and column indices in $S$. It should be noted that $\prod_{j=1}^{n} \operatorname{det}\left(Y_{D_{j}}^{C_{j}}\right) \neq 0$ if and only if $C \leq D$.

Let $X=\operatorname{diag}\left(x_{1}, \ldots, x_{n}\right)$ be a diagonal matrix, which can be viewed as a linear transformation from $\mathcal{M}_{D}$ to $\mathcal{M}_{D}$ via the $B$-action. The character of $\mathcal{M}_{D}$ is defined as the trace of $X$ :

$$
\operatorname{char}\left(\mathcal{M}_{D}\right)(x)=\operatorname{tr}\left(X: \mathcal{M}_{D} \rightarrow \mathcal{M}_{D}\right) .
$$

The dual character of $\mathcal{M}_{D}$ is the character of the dual module $\mathcal{M}_{D}^{*}$, which is given by

$$
\begin{aligned}
\operatorname{char}^{*}\left(\mathcal{M}_{D}\right)(x) & =\operatorname{tr}\left(X: \mathcal{M}_{D}^{*} \rightarrow \mathcal{M}_{D}^{*}\right) \\
& =\operatorname{char}\left(\mathcal{M}_{D}\right)\left(x_{1}^{-1}, \ldots, x_{n}^{-1}\right) .
\end{aligned}
$$

Two families of flagged Weyl modules are of particular interest. The first one is the flagged Weyl module associated to the Rothe diagram $D(w)$ of a permutation $w$ of $[n]$. In this case, Kraśkiewicz and Pragacz [8,9] showed that the Schubert polynomial $\mathfrak{S}_{w}(x)$ of $w$ is equal to the dual character of $\mathcal{M}_{D(w)}$. The second one is the flagged Weyl module associated to the skyline diagram of a composition $\alpha$, which is the structure that we need for the purpose of this paper.

The skyline diagram $D(\alpha)$ of a composition $\alpha$ is the diagram consisting of the first $\alpha_{i}$ boxes in row $i$. For example, Figure 2.2 illustrates the skyline diagram of $\alpha=(1,2,0,1)$.

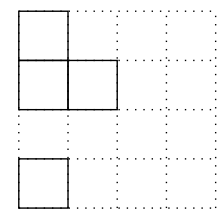

Figure 2.2: The skyline diagram of $\alpha=(1,2,0,1)$.

Theorem 2.1 (Demazure [2]). Let $D(\alpha)$ be the skyline diagram of a composition $\alpha$. Then

$$
\kappa_{\alpha}(x)=\operatorname{char}^{*}\left(\mathcal{M}_{D(\alpha)}\right)(x)
$$

Notice that, for $C \leq D$, the effect of the action of the diagonal matrix $X=$ $\operatorname{diag}\left(x_{1}, \ldots, x_{n}\right)$ on the polynomial $\prod_{j=1}^{n} \operatorname{det}\left(Y_{D_{j}}^{C_{j}}\right)$ is

$$
\prod_{j=1}^{n} \operatorname{det}\left(Y_{D_{j}}^{C_{j}}\right) \cdot X=\prod_{j=1}^{n} \prod_{i \in C_{j}} x_{i}^{-1} \cdot \prod_{j=1}^{n} \operatorname{det}\left(Y_{D_{j}}^{C_{j}}\right) .
$$

This implies that the polynomial $\prod_{j=1}^{n} \operatorname{det}\left(Y_{D_{j}}^{C_{j}}\right)$ is an eigenvector of $X$ with eigenvalue

$$
\prod_{j=1 \in x_{j}}^{n} \prod_{x_{i}^{-1}}
$$


For any diagram $C=\left(C_{1}, \ldots, C_{n}\right)$, let

$$
x^{C}=\prod_{j=1}^{n} \prod_{i \in C_{j}} x_{i} .
$$

Therefore, the set of monomials appearing in the dual character $\operatorname{char}^{*}\left(\mathcal{M}_{D}\right)(x)$ is exactly $\left\{x^{C}: C \leq D\right\}$. Restricting $D$ to a skyline diagram $D(\alpha)$ and combining Theorem 2.1], we are given the following description of monomials appearing in $\kappa_{\alpha}(x)$.

Theorem 2.2. The set of monomials appearing in $\kappa_{\alpha}(x)$ is

$$
\left\{x^{C}: C \leq D(\alpha)\right\}
$$

\section{Proof of Theorem 1.1}

To provide a proof of Theorem 1.1, we first characterize the monomials appearing in $\kappa_{\alpha}(x)$ in terms of certain fillings of skyline diagrams.

Let $D=\left(D_{1}, D_{2}, \ldots, D_{n}\right)$ be a diagram of $[n]^{2}$. A filling $F$ of $D$ is an assignment of positive integers into the boxes of $D$. A filling $F$ is called column-strict if the integers in each column of $F$ are distinct, and $F$ is called flagged if for each box in row $i$, the integer assigned in it does not exceed $i$. We denote by $\mathcal{F}(D)$ the set of column-strict flagged fillings of $D$. We also define $\mathcal{F}_{\leq}(D)$ to be the subset of $\mathcal{F}(D)$ consisting of the fillings $F \in \mathcal{F}(D)$ such that the integers in each column of $F$ are increasing from top to bottom. For example, Figure 3.3(a) is a filling in $\mathcal{F}(D)$, while Figure 3.3(b) is a filling in $\mathcal{F}_{\leq}(D)$. For a filling $F \in \mathcal{F}(D)$, write $\operatorname{wt}(F)=\left(v_{1}, v_{2}, \ldots, v_{n}\right)$ to be the weight of $F$, where $v_{i}$ is the number of appearances of $i$ in $F$.

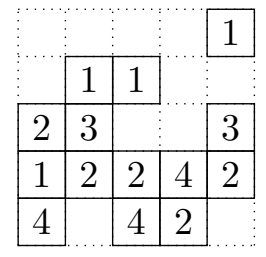

(a)

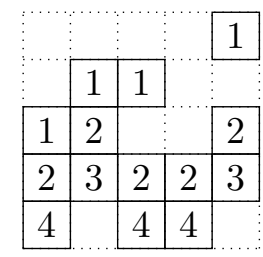

(b)

Figure 3.3: Two fillings of a diagram $D$.

Theorem 3.1. The set of monomials appearing in $\kappa_{\alpha}(x)$ is

$$
\left\{x^{\mathrm{wt}(F)}: F \in \mathcal{F}(D(\alpha))\right\} \text {. }
$$

Proof. For any diagram $D$, there is a direct bijection between $\mathcal{F}_{\leq}(D)$ and the set $\{C: C \leq$ $D\}$. To be specific, given a filling $F \in \mathcal{F}_{\leq}(D)$, let $C=\left(C_{1}, C_{2}, \ldots, C_{n}\right)$ be the diagram such that for $1 \leq j \leq n, C_{j}$ is the set of integers filled in the $j$-th column of $F$. Therefore, 
by Theorem 2.2 , the set of monomials appearing in $\kappa_{\alpha}(x)$ is $\left\{x^{\mathrm{wt}(F)}: F \in \mathcal{F}_{\leq}(D(\alpha))\right\}$. We conclude the proof by showing that for any diagram $D$,

$$
\left\{x^{\mathrm{wt}(F)}: F \in \mathcal{F}_{\leq}(D)\right\}=\left\{x^{\mathrm{wt}(F)}: F \in \mathcal{F}(D)\right\}
$$

It follows from $\mathcal{F}_{\leq}(D) \subseteq \mathcal{F}(D)$ that $\left\{x^{\mathrm{wt}(F)}: F \in \mathcal{F}_{\leq}(D)\right\} \subseteq\left\{x^{\mathrm{wt}(F)}: F \in \mathcal{F}(D)\right\}$.

Now we verify the reverse inclusion. Given a filling $F \in \mathcal{F}(D)$, let $F^{\prime}$ be a filling obtained from $F$ by resorting the integers in each column increasingly from top to bottom. For example, if $F$ is the filling in Figure 3.3(a), then $F^{\prime}$ is the filling in Figure 3.3(b). By [3, Proposition 3.5], $F^{\prime}$ belongs to $\mathcal{F}_{\leq}(D)$. Since $F$ and $F^{\prime}$ have the same weight, we see that

$$
\left\{x^{\mathrm{wt}(F)}: F \in \mathcal{F}(D)\right\} \subseteq\left\{x^{\mathrm{wt}(F)}: F \in \mathcal{F}_{\leq}(D)\right\} .
$$

This proves (3.1), and so the proof is complete.

We now prove the necessity of Theorem 1.1 .

Theorem 3.2. If $\beta$ is an exponent vector of $\kappa_{\alpha}(x)$, then $\beta \leq_{\kappa} \alpha$.

To prove Theorem 3.2, we introduce an operation, called optimization, on the fillings of $\mathcal{F}(D)$. Given a filling $F \in \mathcal{F}(D)$, the optimization of $F$, denoted opt $(F)$, is obtained by rearranging the integers in each column of $F$ as follows. Write $D=\left(D_{1}, \ldots, D_{n}\right)$. For $1 \leq m \leq n$, let $C_{m}$ denote the set of integers filled in the $m$-th column $F_{m}$ of $F$. Suppose that

$$
C_{m} \cap D_{m}=\left\{i_{1}<i_{2}<\cdots<i_{k}\right\}
$$

Let us construct the $m$-th column $\bar{F}_{m}$ of opt $(F)$. Set $\bar{F}_{m}^{(0)}=F_{m}$. For $1 \leq r \leq k, \bar{F}_{m}^{(r)}$ is obtained from $\bar{F}_{m}^{(r-1)}$ as below. If $i_{r}$ is filled in the box $\left(i_{r}, m\right)$ of $\bar{F}_{m}^{(r-1)}$, then let $\bar{F}_{m}^{(r)}=\bar{F}_{m}^{(r-1)}$. Otherwise, $i_{r}$ is filled in a box $(t, m)$ of $\bar{F}_{m}^{(r-1)}$ with $t>i_{r}$. Let $\bar{F}_{m}^{(r)}$ be obtained from $\bar{F}_{m}^{(r-1)}$ by interchanging $i_{r}$ and the integer filled in the box $(t, m)$. Define $\bar{F}_{m}=\bar{F}_{m}^{(k)}$. By the above construction, each column $\bar{F}_{m}^{(r)}$ satisfies the flag constraint. Hence $\operatorname{opt}(F)$ is a filling belonging to $\mathcal{F}(D)$. Moreover, opt $(F)$ has the same weight as $F$.

For example, if $F$ is the filling in Figure 3.3(a), then opt $(F)$ is the filling as given in Figure 3.4.

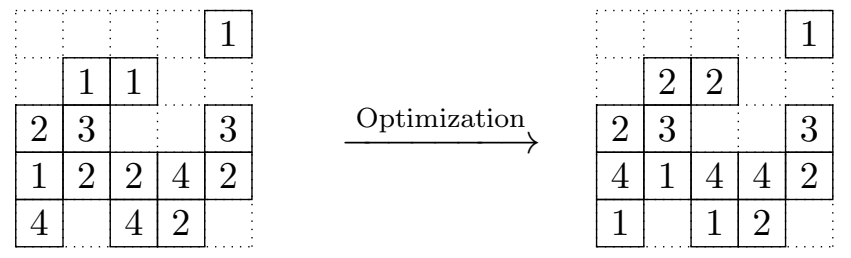

Figure 3.4: $\operatorname{opt}(F)$ for $F$ being the filling in Figure 3.3)(a).

Using the optimization operation, we have the following lemma, which is crucial to the proof of Theorem 3.2 . 
Lemma 3.3. Let $\alpha=\left(\alpha_{1}, \ldots, \alpha_{n}\right)$ be a composition, and $F$ be a filling in $\mathcal{F}(D(\alpha))$ with $\operatorname{wt}(F) \neq \alpha$. Then there exists a filling $F^{\prime} \in \mathcal{F}(D(\alpha))$ such that $\operatorname{wt}(F)=t_{i, j}\left(\operatorname{wt}\left(F^{\prime}\right)\right)$ or $\operatorname{wt}(F)=m_{i, j}\left(\operatorname{wt}\left(F^{\prime}\right)\right)$.

Proof. We aim to construct a filling $F^{\prime} \in \mathcal{F}(D(\alpha))$ from the optimization opt $(F)$ of $F$ such that $\operatorname{wt}(\operatorname{opt}(F))=t_{i, j}\left(\operatorname{wt}\left(F^{\prime}\right)\right)$ or $\operatorname{wt}(\operatorname{opt}(F))=m_{i, j}\left(\operatorname{wt}\left(F^{\prime}\right)\right)$.

Since $\operatorname{wt}(\operatorname{opt}(F))=\operatorname{wt}(F) \neq \alpha$, there must exist a box of opt $(F)$ that is filled with an integer not equal to its row index. Locate the topmost row of opt $(F)$, say row $j$, which contains an entry not equal to its row index. Suppose that $i$ is the leftmost entry in the $j$-th row of $\operatorname{opt}(F)$ that is not equal to $j$. Since opt $(F)$ satisfies the flag condition, we have $i<j$. Moreover, let $(j, h)$ be the box in row $j$ that contains this leftmost entry $i$. Write wt $(\operatorname{opt}(F))=\left(\beta_{1}, \ldots, \beta_{n}\right)$. The construction of $F^{\prime}$ depends on the relative order of $\beta_{i}$ and $\beta_{j}$.

Case $1 . \beta_{i} \leq \beta_{j}$. The filling $F^{\prime}$ is obtained from opt $(F)$ by replacing the entry $i$ in the box $(j, h)$ with $j$. We explain that $F^{\prime}$ is a filling in $\mathcal{F}(D(\alpha))$. Clearly, $F^{\prime}$ satisfies the flag condition. We still need to verify that $F^{\prime}$ is column strict. By the construction of $\operatorname{opt}(F)$, there is no integer in the $h$-th column of opt $(F)$ that is equal to $j$, since otherwise the box $(j, h)$ of $\operatorname{opt}(F)$ would be filled with $j$. This implies that $F^{\prime}$ is column strict, and thus $F^{\prime} \in \mathcal{F}(D(\alpha))$. By the construction of $F^{\prime}$, we see that

$$
\beta=m_{i, j}\left(\mathrm{wt}\left(F^{\prime}\right)\right)
$$

For example, Figure 3.5 is an illustration of the construction of $F^{\prime}$ in Case 1, where the integer in boldface signifies the integer $i$ that is changed to $j$.

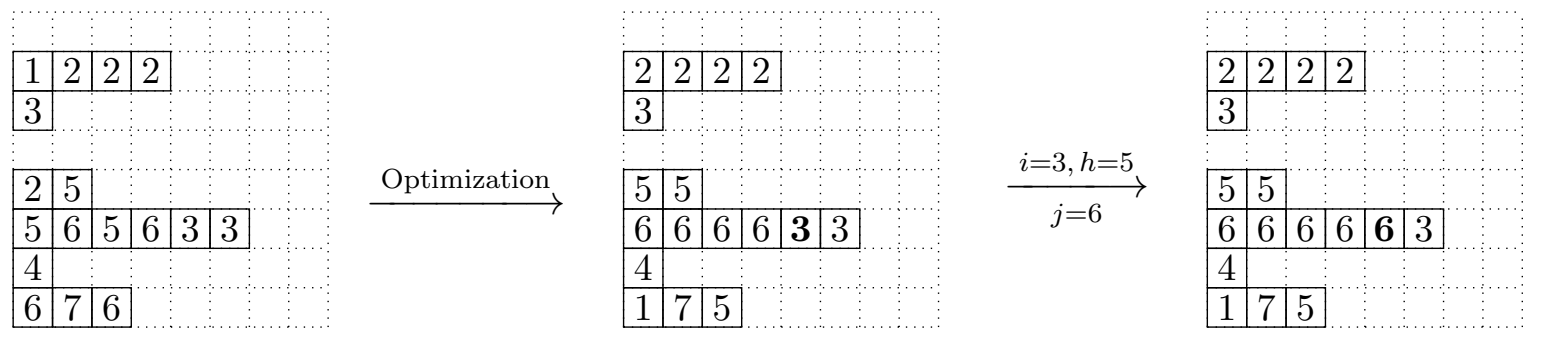

Figure 3.5: An example to illustrate Case 1.

Case 2. $\beta_{i}>\beta_{j}$. The filling $F^{\prime}$ is obtained from opt $(F)$ according to the following rule. For $1 \leq m \leq n$, let $F_{m}$ be the $m$-th column of opt $(F)$, and $F_{m}^{\prime}$ be the $m$-th column of $F^{\prime}$.

(i) If $F_{m}$ contains both $i$ and $j$ or contains neither $i$ nor $j$, then let $F_{m}^{\prime}=F_{m}$;

(ii) If $F_{m}$ contains only $i$, then $F_{m}^{\prime}$ is obtained from $F_{m}$ by replacing $i$ with $j$;

(iii) If $F_{m}$ contains only $j$, then $F_{m}^{\prime}$ is obtained from $F_{m}$ by replacing $j$ with $i$.

For example, Figure 3.6 is an illustration of the construction of $F^{\prime}$ in Case 2, where the integers in boldface signify the integers $i$ and $j$ that are interchanged. 


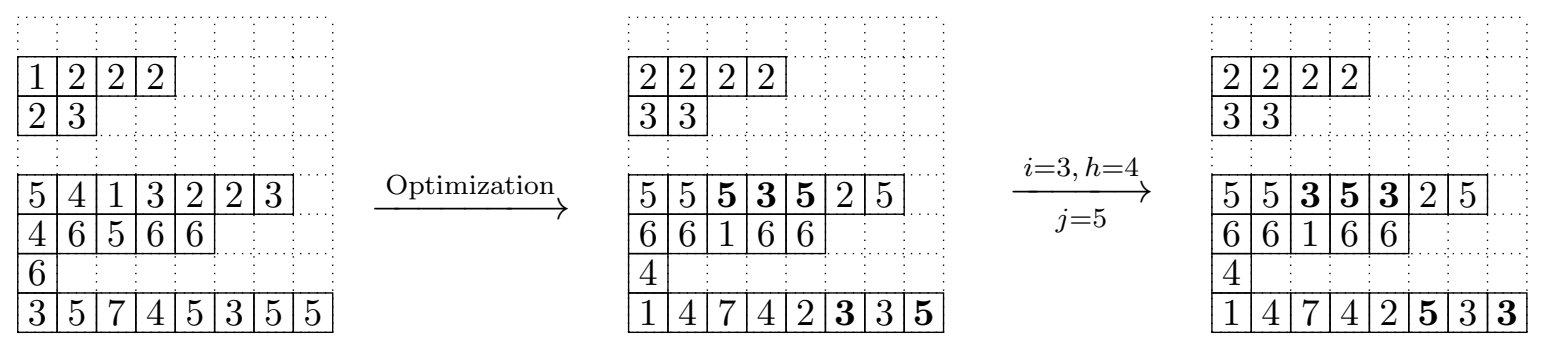

Figure 3.6: An example to illustrate Case 2.

It remains to show that $F^{\prime}$ belongs to $\mathcal{F}(D(\alpha))$. By the above constructions, $F_{m}^{\prime}$ is column strict. We need to show that $F_{m}^{\prime}$ satisfies the flag condition. This is evident if $F_{m}^{\prime}$ is obtained from $F_{m}$ by applying (i) or (iii). We next prove the case when $F_{m}^{\prime}$ is obtained from $F_{m}$ by applying (ii). Suppose that the entry $i$ in $F_{m}$ lies in the box $(l, m)$.

We first assert that $\alpha_{i}<h$. Suppose otherwise that $\alpha_{i} \geq h$. Then the box $(i, h)$ belongs to $D(\alpha)$. By the choice of the index $j$, the box $(i, h)$ is filled with $i$. Since the box $(j, h)$ is also filled with $i$, the column $F_{h}$ of $F$ is not column strict, leading to a contradiction. This verifies the assertion.

Based on the above assertion, we claim that $l \geq j$. Suppose otherwise that $l<j$. Again, by the choice of the index $j$, we must have $l=i$. Since $(l, m)$ lies in the $i$-th row, we have $m \leq \alpha_{i}$, which, together with the above assertion that $\alpha_{i}<h$, implies $m<h$. Note that each box in row $j$ of $F$ that is to the left of $(j, h)$ is filled with $j$. This implies that the column $F_{m}$ contains both $i$ and $j$, which contradicts the fact that $F_{m}$ contains only $i$. Hence the claim is true.

By the above claim, we see that $F_{m}^{\prime}$ satisfies the flag constraint, and so $F^{\prime}$ belongs to $\mathcal{F}(D(\alpha))$. Moreover, it is clear that $\beta=t_{i, j}\left(\operatorname{wt}\left(F^{\prime}\right)\right)$. This completes the proof.

Based on Lemma 3.3, we can now provide a proof of Theorem 3.2.

Proof of Theorem 3.2. Let $\beta$ be an exponent vector of $\kappa_{\alpha}(x)$. We aim to show that $\beta \leq_{\kappa} \alpha$. By Theorem 3.1, there exists a filling $F \in \mathcal{F}(D(\alpha))$ with $\operatorname{wt}(F)=\beta$. The theorem is trivial in the case $\beta=\alpha$. We now consider the case $\beta \neq \alpha$.

By Lemma 3.3, there exists a filling $F^{\prime}$ in $\mathcal{F}(D(\alpha))$ such that $\operatorname{wt}(F)=t_{i, j}\left(\operatorname{wt}\left(F^{\prime}\right)\right)$ or $\operatorname{wt}(F)=m_{i, j}\left(\operatorname{wt}\left(F^{\prime}\right)\right)$. Notice that $\mathrm{wt}(F)>_{\text {lex }} \operatorname{wt}\left(F^{\prime}\right)$, where $\geq_{\text {lex }}$ is the lexicographic order on compositions. If $\operatorname{wt}\left(F^{\prime}\right) \neq \alpha$, then we can again invoke Lemma 3.3 to find a filling $F^{\prime \prime} \in \mathcal{F}(D(\alpha))$ such that $\operatorname{wt}\left(F^{\prime}\right)=t_{i, j}\left(\operatorname{wt}\left(F^{\prime \prime}\right)\right)$ or $\operatorname{wt}\left(F^{\prime}\right)=m_{i, j}\left(\operatorname{wt}\left(F^{\prime \prime}\right)\right)$. Continuing this procedure, we can eventually arrive at the (unique) filling $F_{0} \in \mathcal{F}(D(\alpha))$ with wt $\left(F_{0}\right)=\alpha$. Here, $F_{0}$ is the filling such that each box of $D(\alpha)$ is filled with its row index. Hence $\beta=\operatorname{wt}(F)$ can be generated from $\alpha=\operatorname{wt}\left(F_{0}\right)$ by applying a sequence of moves $t_{i, j}$ and $m_{i, j}$, implying that $\beta \leq_{\kappa} \alpha$. This completes the proof.

We lastly prove the sufficiency of Theorem 1.1 .

Theorem 3.4. If $\beta \leq_{\kappa} \alpha$, then $\beta$ is an exponent vector of $\kappa_{\alpha}(x)$.

Proof. Let $\beta=\beta^{(0)}, \beta^{(1)}, \ldots, \beta^{(k)}=\alpha$ be a sequence of compositions such that for $0 \leq t \leq k-1, \beta^{(t)}$ is obtained from $\beta^{(t+1)}$ by applying $t_{i, j}$ or $m_{i, j}$. The proof that $\beta$ is 
an exponent vector of $\kappa_{\alpha}(x)$ is by induction on $k$. The case for $k=0$ (namely, $\beta=\alpha$ ) is trivial. We next consider the case $k>0$.

By hypothesis, $\beta^{(1)}$ is an exponent vector of $\kappa_{\alpha}(x)$. It follows from Theorem 3.1 that there is a filling $F$ in $\mathcal{F}(D(\alpha))$ such that $\operatorname{wt}(F)=\beta^{(1)}$. To complete proof, it suffices to construct a filling $F^{\prime}$ in $\mathcal{F}(D(\alpha))$ such that $\operatorname{wt}\left(F^{\prime}\right)=\beta$. Write $\beta^{(1)}=\left(v_{1}, \ldots, v_{n}\right)$. We have two cases.

Case 1. $\beta=t_{i, j}\left(\beta^{(1)}\right)$. In this case, $v_{i}<v_{j}$. Suppose that there are $c_{1}$ columns of $F$ that contain $i$ but do not contain $j$. Then there are $c_{1}+v_{j}-v_{i}$ columns of $F$ that contain $j$ but do not contain $i$. Choose any $v_{j}-v_{i}$ such columns, and let $F^{\prime}$ be obtained from $F$ by replacing $j$ in each of these columns with $i$. It is readily seen that $F$ belongs to $\mathcal{F}(D(\alpha))$ with $\operatorname{wt}\left(F^{\prime}\right)=\beta$.

Case 2. $\beta=m_{i, j}\left(\beta^{(1)}\right)$. In this case, $v_{i}<v_{j}-1$. Hence there is at least one column of $F$ that contains $j$ but does not contain $i$. Choose one such column, and let $F^{\prime}$ be obtained from $F$ by replacing $j$ in this column with $i$. It is also clear that $F$ belongs to $\mathcal{F}(D(\alpha))$ with $\operatorname{wt}\left(F^{\prime}\right)=\beta$. This completes the proof.

Acknowledgments. This work was supported by the 973 Project and the National Science Foundation of China (Grant No. 11971250).

\section{References}

[1] M. Demazure, Désingularisation des variétés de Schubert généralisées, Ann. Sci. École Norm. Sup. 7 (1974), 53-88.

[2] M. Demazure, Une nouvelle formule des caractéres, Bull. Sci. Math. (2) 98 (1974), $163-172$.

[3] N.J.Y. Fan and P.L. Guo, Vertices of Schubitopes, arXiv:1910.02816.

[4] A. Fink, K. Mészáros and A. St. Dizier, Schubert polynomials as integer point transforms of generalized permutahedra, Adv. Math. 332 (2018), 465-475.

[5] J. Haglund, K. Luoto, S. Mason and S. van Willigenburg, Refinements of the Littlewood-Richardson rule, Trans. Amer. Math. Soc. 363 (2011), 1665-1686.

[6] B. Ion, Nonsymmetric Macdonald polynomials and Demazure characters, Duke Math. J. 116 (2003), 299-318.

[7] Y. Kodama and L. Williams, The full Kostant-Toda hierarchy on the positive flag variety, Comm. Math. Phys. 335 (2015), 247-283.

[8] W. Kraśkiewicz and P. Pragacz, Foncteurs de Schubert, C. R. Acad. Sci. Paris Sér. I Math. 304 (1987), 209-211.

[9] W. Kraśkiewicz and P. Pragacz, Schubert functors and Schubert polynomials, European J. Combin. 25 (2004),1327-1344. 
[10] A. Lascoux and M.-P. Schützenberger, Keys \& standard bases, Invariant Theory and Tableaux (Minneapolis, MN, 1988), 125-144, IMA Vol. Math. Appl., 19, Springer, New York, 1990.

[11] A. Lascoux and M.-P. Schützenberger, Tableaux and non-commuative Schubert polynomials, Func. Anal. Appl. 23 (1989), 63-64.

[12] P. Magyar, Schubert polynomials and Bott-Samelson varieties, Comment. Math. Helv. 73 (1998), 603-636.

[13] S. Mason, An explicit construction of type $A$ Demazure atoms, J. Algebraic Combin. 29 (2009), 295-313.

[14] C. Monical, N. Tokcan and A. Yong, Newton polytopes in algebraic combinatorics, Selecta Math. (N.S.) 25 (2019), no. 5, Paper No. 66.

[15] R. Rado, An inequality, J. Lond. Math. Soc. 27 (1952), 1-6.

[16] V. Reiner and M. Shimozono, Key polynomials and a flagged Littlewood-Richardson rule, J. Combin. Theory Ser. A 70 (1995), 107-143.

[17] E. Tsukerman and L. Williams, Bruhat interval polytopes, Adv. Math. 285 (2015), 766-810.

Neil J.Y. Fan, Department of Mathematics, Sichuan University, Chengdu 610064, P.R. CHINA. Email address: fan@scu.edu.cn.

Peter L. Guo, Center for Combinatorics, Nankai University, Tianjin 300071, P.R. CHINA. Email address: lguo@nankai.edu.cn.

Simon C.Y. Peng, Center for Applied Mathematics, Tianjin University, Tianjin 300072, P.R. ChinA. Email address: pcy@tju.edu.cn.

Sophie C.C. Sun, Department of Mathematics, University of FinanCe and Economics, TIANJIN 300222, P.R. ChINA. Email address: suncongcong@mail.nankai.edu.cn. 